\title{
THE IDENTIFICATION METHOD OF 6-7 YEAR-OLD-CHILDREN’S «DUOVECTOR» GIFTEDNESS IN THE ART-CREATIVITY PROCESS
}

\author{
(C) 2018 \\ Kalinina Larisa Yuryevna, candidate of pedagogical sciences, leading researcher of Research Department \\ Ivanov Dmitriy Victorovich, candidate of psychological sciences, \\ associate professor of Pedagogy and Psychology Department \\ Samara State University of Social Sciences and Education (Samara, Russian Federation)
}

\begin{abstract}
The paper deals with one of the aspects of early identification of giftedness: the establishment of the relationship between its types. The authors see a solution of the problem in the development and validation of the methodology based on the integrated modern scientific knowledge - psychological, pedagogical and art criticism, in the field of contemporary art. This technique is expected to meet the conditions of efficiency and accessibility in the application of teachers working with children. Clarifications have been made to the basic concept of «giftedness» for the paper. The authors propose a term describing the interrelated manifestation of two types of giftedness - «duovector» talent. The method is aimed at finding hidden signs of «duovector» giftedness: musical mathematics, in the field of fine art and sports, musical and linguistic. The basis of this approach is the idea of the dependence of the frequency and brightness of giftedness manifestations on the conditions, the most important of which is the aesthetic environment enriched with multi-modal material for creativity. At the same time, it is advisable to involve children in accessible and aesthetically valuable works of modern art, in the search for new knowledge in the same ways that adult authors of the XXI century use. Modeling directly perceived creative techniques and forms, the child masters the world, structures it non-linearly, on the principle of creating a rhizome. As a «catalyst» of creative activity, a set of tasks-subtests adapted to the age peculiarities of children is offered. The procedure of the experiment in a specially organized educational environment (an art workshop) is characterized. Plunging into the atmosphere of «fruitful disorder», the child will act freely and directly, engaged in creativity as a game, creates an art product that has value as a marker of his talent. The content of creative tasks is presented, according to the results of work on which the diagnostic card is filled, in turn, which is the basis for the conclusions about the presence of the child's «duovector» talent. At this stage of the study, the authors have prepared a method for validation by comparing it with tests and subtests of other methods. The materials of the paper logically continue the research in the field of finding reference points for the development of individual educational routes of students, preparing them for lifelong learning.

Keywords: giftedness; gifted children; education; creativity of child; synthesis of arts; educational artistic and creative activity; rhizome; senior preschoolers; first-graders; art workshop; individual educational trajectory; contemporary art; art composition.
\end{abstract}

УДК 378.147.001.572:51

DOI $10.24411 / 2309-4370-2018-14309$

Статья поступила в редакцию 19.08.2018

(C) 2018

\section{ВЕКТОРНАЯ ИНТЕРПРЕТАЦИЯ ОЦЕНКИ ОСТАТОЧНЫХ ЗНАНИЙ ОБУЧАЮЩИХСЯ}

\author{
Макаров Сергей Иванович, доктор педагогических наук, \\ заведующий кафедрой высшей математики и экономико-математических методов \\ Севастьянова Светлана Александровна, кандидат педагогических наук, \\ доцент кафедры высшей математики и экономико-математических методов \\ Самарский государственный экономический университет (г. Самара, Российская Федераџия)
}

Аннотация. Контроль остаточных знаний - одна из процедур, используемых в системе образования России для мониторинга качества образования на различных уровнях. Как правило, эта процедура реализуется в форме компьютерного тестирования по изученным ранее дисциплинам. Анализ результатов тестирования дает основание для принятия решений о выполнении коррекционных мероприятий. Это определяет важность задачи достоверного, доступного и информативного представления результатов мониторинга. В статье предложен метод визуализации и интерпретации результатов контроля остаточных знаний, обоснована возможность его использования в целях анализа проблем обучения на индивидуальном и групповом уровнях. Предложено использовать для обработки статистической информации векторную форму представления совокупности данных. В этом случае визуализация результатов контроля имеет вид пространственного вектора или развертки многомерного вектора на плоскость. Обсуждаются возможности визуальной оценки достижения пороговых значений уровня знаний. Приведены примеры использования векторной модели для оценки уровня остаточных знаний по трем и более дисциплинам. Предложен подход к решению вопроса о сопоставимости результатов тестирований, проведенных в различных системах оценивания. Основные выводы и результаты могут быть использованы непосредственно в учебном процессе, в сфере управления образованием, в психолого-педагогической работе.

Ключевые слова: математические методы в педагогике; педагогическая диагностика; результаты тестирования; контроль остаточных знаний; обработка данных мониторинга; оценка знаний; математическая обработка результатов тестирования; визуальная интерпретация оценки знаний; лепестковая диаграмма. 
Одной из нерешенных проблем в образовании является оценка остаточных знаний обучающихся. Она необходима для педагогической диагностики, коррекции образовательного процесса, выбора форм и методов преподавания дисциплин, оценки качества образования, эффективного управления образованием. В большей мере процедура проверки остаточных знаний используется в сфере высшего образования, поскольку эта система имеет больше возможностей для внесения изменений в учебный процесс как на индивидуальном уровне, так и на уровне учебного заведения и системы образования в целом. Результаты контроля остаточных знаний студентов являются одним из факторов, имеющих значение при лицензировании учебного заведения и аккредитации учебных программ.

Контроль остаточных знаний должен обладать признаками целенаправленности, объективности, полноты, адекватности, а также информативности и наглядности представления результатов. Последнее необходимо для того, чтобы по результатам проведенной проверки можно было сделать правильные выводы и при необходимости организовать коррекционный процесс. Количественная оценка, выраженная графическим способом, имеет преимущества в восприятии и анализе перед цифровой формой представления результатов. Визуализация статистических данных в виде диаграмм и графиков является стандартной практикой представления информации в бизнесе, менеджменте, экономике.

В работах педагогов-исследователей отражен современный опыт проведения, анализа и интерпретации оценки знаний обучающихся. Специфика диагностической работы в вузе обсуждается в научных работах Е.К. Артищевой [1]. Глубоко раскрыта тема функционального предназначения и организации тестового контроля знаний [2, с. 121]. В научных трудах [3-7] предлагаются различные способы обработки результатов квалиметрических процедур с целью диагностики и коррекции знаний. Ряд исследований посвящен вопросам представления полученных данных в различных формах [8-13]. Проблемам контроля остаточных знаний посвящены труды О.И. Федяева [14], Е.К. Артищевой [15].

Вместе с тем, при наличии значительного числа публикаций по данной тематике, актуальными остаются проблемы обработки, анализа и интерпретации полученных данных. На основании анализа литературных источников по данной проблеме можно сделать вывод о том, что в большинстве случаев анализ результатов производится по общим статистическим методам: рассчитываются выборочные характеристики, строятся гистограммы, проверяются различные гипотезы, строятся модели и прогнозы и т.д. Эти научные подходы хорошо выполняют свою квалиметрическую функцию, но имеют существенный недостаток: сложность восприятия для неподготовленного пользователя. В данной работе предложен иной подход к представлению результатов.

Методология исследования включает общенаучные и математические методы: теоретический анализ проблемы на основе анализа научной литературы, обобщение опыта для определения путей решения проблемы, сравнение, математическое моделирование, использование фундаментальных основ векторной алгебры для решения прикладных задач.
Поиск новых путей визуального представления результатов контроля, их обработки и анализа приводит к идее использования математических методов в образовательной деятельности. В сложившейся практике процедура проверки остаточных знаний является частью внутреннего или внешнего мониторинга и, как правило, основана на предметном тестировании с определенным лагом по времени. Количественная оценка результатов тестирования имеет форму упорядоченной совокупности данных, а следовательно, может быть представлена в векторной форме и проанализирована на основе векторного критерия. Компоненты вектора могут быть сгенерированы на основе текущей успеваемости обучающихся или по результатам более позднего тестирования.

В таком случае проблема оценки остаточных знаний может быть сведена к задаче построения отношения, преобразующего каждый результат тестирования по одной дисциплине в некоторое вполне упорядоченное множество [16], как, например, множество $[0,100]$. Причем отношение должно быть построено таким образом, чтобы качественные характеристики возрастали с увеличением значения каждого критерия. Это позволит геометрически соотнести результаты.

Будем считать, что обучение в образовательном учреждении длится $k$ лет и каждый год проверка остаточных знаний проводится по $n_{r}$ предметам, $1 \leq r \leq k$ и $\sum_{r=1}^{k} n_{r}=n$. Считаем, что контроль осуществляется в форме тестирования по 100-балльной шкале. Количество баллов, набранных обучаемым при контроле текущей успеваемости прошедшего периода и при проверке остаточных знаний по $i$-той дисциплине на $j$-том году обучения, обозначим $x_{i j}^{0}$ и $x_{i j}$ соответственно.

Таким образом, всей совокупности результатов проверки текущей успеваемости и остаточных знаний по всем дисциплинам ставятся в соответствие упорядоченные кортежи из $n$ чисел - векторы мониторинга текущей успеваемости и остаточных знаний.

$$
\begin{gathered}
\bar{X}^{0}\left(x_{11}^{0}, x_{12}^{0}, \ldots, x_{1 n_{1}}^{0} ; \ldots ; x_{k 1}^{0}, x_{k 2}^{0}, \ldots, x_{k n_{k}}^{0}\right) \text { и } \\
\bar{X}\left(x_{11}, x_{12}, \ldots, x_{1 n_{1}} ; \ldots ; x_{k 1}, x_{k 2}, \ldots, x_{k n_{k}}\right) .
\end{gathered}
$$

Эти объекты с математической точки зрения представляют собой векторы в n-мерном пространстве, с семантической точки зрения они являются оценкой текущей успеваемости и остаточных знаний. Эти вектора расположены в n-мерном кубе:

$$
\mathrm{C}=[0,100] \times[0,100] \times \ldots \times[0,100] .
$$

Например, вектор $\bar{X}_{I}-n$-мерный вектор, все координаты которого равны 100, соответствует «идеальному обучающемуся», набравшему максимальное число баллов по всем дисциплинам.

Для визуальной и количественной оценки соответствия уровня знаний заданным минимальным требованиям можно предложить следующий подход. 
В данном кубе можно выделить два подмножества $-\mathrm{C}_{\text {T и }} \mathrm{C}_{\mathrm{N}}$

$$
\mathrm{CT} \cup \mathrm{CN}=\mathrm{C},
$$

где $\mathrm{C}_{\mathrm{T}}=[50,100] \times[50,100] \times \ldots \times[50,100]-\mathrm{n}$ мерный куб, в который попали концы векторов, все координаты которых удовлетворяют минимальным требованиям, предъявляемым к текущим и остаточным знаниям обучающегося; $\mathrm{C}_{\mathrm{N}}=\mathrm{C} / \mathrm{C}_{\mathrm{T}}$ - дополнение $\mathrm{C}_{\mathrm{T}}$ до множества С. В множество $\mathrm{C}_{\mathrm{N}}$ попадут концы векторов, у которых хотя бы одна координата не удовлетворяет минимальным требованиям, предъявляемым к текущим и остаточным знаниям обучающегося.

На первом году обучения в вузе мониторинг остаточных знаний представляет собой оценку начального уровня знаний студента при поступлении в вуз после окончания школы. Тестовые задания должны соответствовать школьной программе и содержанию единого государственного экзамена, но могут быть дополнены и другими вопросами. Начальный минимально допустимый уровень устанавливается на основе предположений о необходимом для успешного обучения объеме усвоенных знаний. Полученные в результате тестирования числовые данные визуализируются в векторном виде.

На рисунке 1 показана векторная интерпретация результатов тестирования студентов первого года обучения при выборе двух дисциплин тестирования. Здесь области $\mathrm{C}$ и $\mathrm{C}_{\mathrm{T}}$ множества двухмерных точек возможных и допустимых результатов тестирования соответственно. Вектор (1) иллюстрирует уровень $\bar{X}_{I}$ с «идеальными характеристиками», вектор (2) визуализирует результаты, не удовлетворяющие минимальным требованиям.

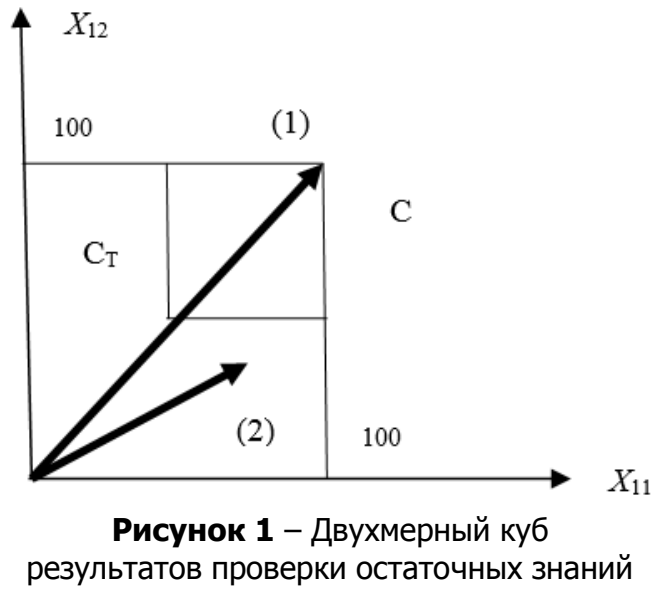

Если количество дисциплин для мониторинга знаний превосходит три $(n>3)$, то построить куб и векторы результатов проверки в $n$-мерном пространстве не представляется возможным. Однако существует прием, позволяющий работать с $n$-мерными векторами графически, используя развертку вектора на плоскость в виде лепестковой диаграммы. Рассмотрим этот прием на примере сравнительного анализа векторов текущей успеваемости и остаточных знаний, приобретенных на первом году обучения при условии, что количество дисциплин мониторинга равно пяти. Тестирование проводилось на втором и третьем году обучения.

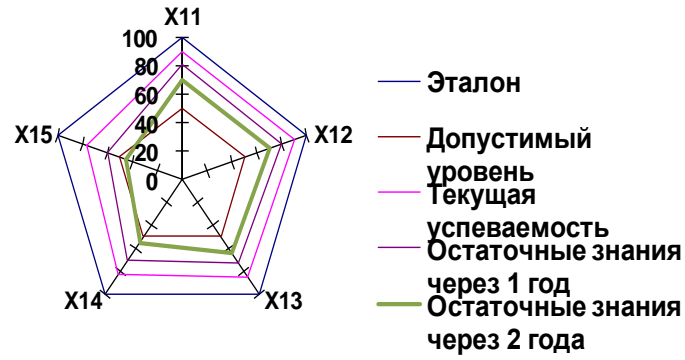

Рисунок 2 - Сравнительный анализ векторов текущей успеваемости и остаточных знаний

На приведенной диаграмме внешняя ломаная линия изображает «идеальный уровень» знаний респондента и соответствует максимально возможному количеству баллов по каждой из дисциплин тестирования. Внутренняя линия соответствует уровню минимальных допустимых требований по каждой из дисциплин и является своего рода барьером, нижним пороговым значением. Другие замкнутые линии диаграммы иллюстрируют результаты контроля текущей успеваемости (промежуточного тестирования, экзамена, зачета) и остаточных знаний через год и через два года после изучения данных дисциплин. Из приведенного рисунка видно, что уровень остаточных знаний убывает с возрастанием времени и по некоторым учебным предметам может опуститься ниже допустимого уровня, как это случилось с дисциплиной $X_{15}$.

Описанный способ визуализации результатов мониторинга текущей успеваемости и остаточных знаний может быть применен не только индивидуально к каждому обучающемуся, но и к однородным группам обучающихся по одинаковым образовательным программам. Для этого формируется вектор $\bar{X}$ group, координаты которого равны средним арифметическим координат векторов мониторинга всех обучающихся.

$$
\bar{X} \text { group }=\frac{1}{m} \sum_{i=1}^{m} \bar{X}_{i},
$$

где $m$ - количество обучающихся в группе, $\bar{X}_{i}$ вектор мониторинга каждого обучающегося.

Представление результатов мониторинга в векторном виде позволит визуально определить проблемные позиции и на основании их анализа и более детального изучения организовать мероприятия по коррекции ситуации или внести изменения в организацию учебного процесса в перспективе.

Векторная интерпретация оценок может быть использована также, например, в психолого-педагогической работе по профессиональной ориентации учащихся. Один из аспектов этой работы - выявление научных и практических предпочтений учащихся в будущей профессии, доминирующей склонности к гуманитарным или естественным наукам и т.д. Для реализации этой задачи по результатам тестирования строятся векторные диаграммы. Приведем пример использования данного подхода при выборе учеником направления обучения. Если количество изучаемых дисциплин в учебном заведении равно $n$, то часть из них $q$ составляют дисциплины социальногуманитарной направленности и $n-q$ дисциплин 
имеют естественнонаучную направленность. Следовательно, $n$-мерный куб:

$$
\mathrm{C}=[0,100] \times[0,100] \times \ldots \times[0,100]
$$

можно представить в виде произведения кубов размерности $q$ и $n-q$ :

$$
\mathrm{C}=\mathrm{C}_{q} \times \mathrm{C}_{n-q} .
$$

Используя проекции векторов мониторинга текущей успеваемости и остаточных знаний на соответствующие пространства размерности $q$ и $n-q$, можно получить векторы мониторинга социальногуманитарной и естественнонаучной направленности, по результатам изучения которых можно сделать выводы о наклонностях обучающегося, а также об уровне преподавания дисциплин соответствующего цикла в данном учебном заведении.

Если векторы мониторинга текущей успеваемости и остаточных знаний были построены в разных масштабах или в различных балльных системах, то для сопоставимости результатов можно эти векторы пронормировать. Выполним нормирование векторов мониторинга $\bar{X}^{0}$ и $\bar{X}$. Для этого разделим их координаты на длины векторов. В результате будут получены векторы тех же направлений единичной длины $\bar{X}_{N}^{0}$ и $\bar{X}_{N}$, концы которых лежат на единичной сфере с центром в начале координат.

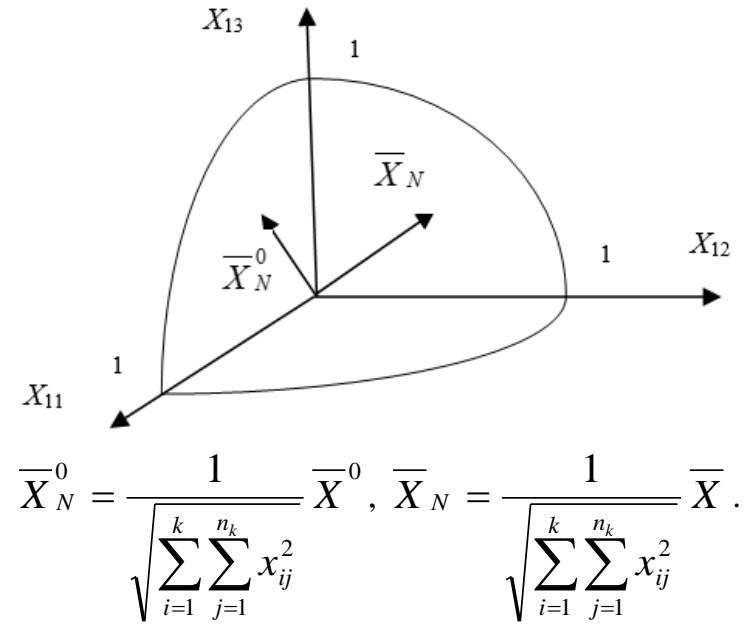

Координаты векторов $\bar{X}_{N}^{0}$ и $\bar{X}_{N}$ равны направляющим косинусам $\cos \alpha_{i}, i=\overline{1, n}$ направлений векторов $\bar{X}^{0}$ и $\bar{X}$. Используя направляющие косинусы можно сопоставлять результаты проверки текущей успеваемости и остаточных знаний одного обучаемого. Чем больше вектор наклонен к одной из осей, тем больше соответствующий направляющий косинус и тем успешнее обучаемый в овладении соответствующей дисциплины.

Предложенный в настоящей работе векторный способ интерпретации результатов мониторинга знаний позволяет визуализировать данные, наглядно представить ситуацию, определить проблемные зоны. Описаны возможные области применения данного способа в квалиметрическом обеспечении мониторинга образования, профориентационной работе, индивидуальной учебной деятельности. Векторный подход открывает новые перспективы использования математических методов в управлении образованием.

\section{Список литературы:}

1. Артищева Е.К. Педагогическая диагностика как основа системы коррекции знаний // Образовательные технологии. 2015. № 3. С. 85-103.

2. Слепухин А.В. Использование новых информационных технологий для контроля и коррекции знаний учащихся по математике: дис. ... канд. пед. наук: 13.00.01. Екатеринбург, 1999. 159 с.

3. Субетто А.И., Чернова Ю.К., Горшенина М.В. Квалиметрическое обеспечение управленческих процессов. СПб.: Изд-во «Астерион», 2004. 278 с.

4. Крашенинникова Ю.В. Статистическая обработка результатов тестирования по высшей математике // Вестник Псковского государственного университета. Серия: Естественные и физико-математические науки. 2012. № 1. С. 116-122.

5. Гуменникова Ю.В., Рябинова Е.Н., Черницына Р.Н. Статистическая обработка результатов тестирования студентов // Вестник Самарского государственного технического университета. Серия: психолого-педагогические науки. 2015. № 3 (27). С. 78-87.

6. Карпинский В.Б. Современные математические методы обработки результатов педагогического тестирования // Альманах современной науки и образования. 2012. № 2. С. 82-84.

7. Шестова Е.А. Разработка моделей и методов анализа и обработки результатов тестирования знаний // Известия ЮФУ. Серия: Технические науки. 2012. № 2 (127). С. 146-152.

8. Ким В.С. Матричное представление результатов тестирования // Вестник Московского государственного областного университета. Серия: Педагогика. 2012. № 4. С. 114-120.

9. Лобова Т.В., Ткачев А.Н. Адаптивная нечеткая процедура интерпретации результатов // Известия высших учебных заведений. Северо-Кавказский регион. Серия: Технические науки. 2014. № 5 (180). C. 102-105.

10. Юрьев Г.А. Математическая модель интерпретации результатов компьютерного тестирования с использованием марковских сетей: дис. ... канд. физ.-мат. наук: 05.13.18, 05.13.01. М., 2013. 108 с.

11. Кремер Н.Ш. Диагностирование и прогнозирование уровня математической подготовки студентов // Современная математика и концепции инновационного математического образования. 2016. Т. 3, № 1. C. $263-265$.

12. Мельникова Н.Н., Щелокова Е.Г. Карьерная направленность: векторная модель диагностики и интерпретации // European Social Science Journal. 2012. № 2 (18). С. 270-277.

13. Севастьянова С.А. Формирование профессиональных математических компетенций у студентов экономических вузов: дис. ... канд. пед. наук: 13.00.08. Самара, 2006. 237 с.

14. Федяев О.И. Прогнозирование остаточных знаний студентов по отдельным дисциплинам с помощью нейронных сетей // Известия ЮФУ. Серия: Технические науки. 2016. № 7 (180). С. 122-136.

15. Артищева Е.К., Брызгалова С.И., Гриценко В.А. Фоновый уровень знаний: сущность, анализ, оценка усвоения: монография. Калининград: Изд-во БФУ им. И. Канта, 2013. 184 с.

16. Макаров С.И. Методические основы создания и применения образовательных электронных изданий (на примере курса математики): дис. ... д-ра пед. наук: 13.00.02. М., 2003. 242 с. 


\section{VECTOR INTERPRETATION OF STUDENTS' RESIDUAL KNOWLEDGE ASSESSMENT}

(C) 2018

Makarov Sergey Ivanovich, doctor of pedagogical sciences, professor, head of Higher Mathematics and Economic-Mathematical Methods Department

Sevastyanova Svetlana Aleksandrovna, candidate of pedagogical sciences, associate professor of Higher Mathematics and Economic-Mathematical Methods Department Samara State University of Economics (Samara, Russian Federation)

Abstract. Residual knowledge assessment is one of the procedures used in the Russian education system to monitor the quality of education at various levels. Typically, this procedure is considered to be a computer testing of the previously learned disciplines. The analysis of test results gives grounds for making necessary decisions. This determines the importance of reliable, accessible and informative presentation of monitoring results. The paper contains a method of visualization and interpretation of residual knowledge assessment results. The authors think that it is possible to use this method for analyzing the problems of training at the individual and group levels. For statistical information processing it is offered to use a vector form of data presentation. The paper contains examples of using a vector model for estimating the level of residual knowledge in three or more disciplines. The authors propose an approach that solves the problem of test results comparability carried out in various estimation systems. The main conclusions and results can be used directly in the educational process, in the field of education management, in psychological and pedagogical work.

Keywords: mathematical methods in Pedagogy; pedagogical diagnostics; test results; control of residual knowledge; processing of monitoring data; knowledge assessment; mathematical processing of test results; visual interpretation of knowledge assessment; petal diagram.

УДК 371.3:378:811.112.2

DOI $10.24411 / 2309-4370-2018-14310$

Статья поступила в редакцию 30.09.2018

\section{ИЗ ОПЫТА ОБУЧЕНИЯ ДЕЛОВОМУ ОБЩЕНИЮ НА ИНОСТРАННОМ ЯЗЫКЕ В МАГИСТРАТУРЕ ЖЕЛЕЗНОДОРОЖНОГО ВУЗА}

(C) 2018

Одарюк Ирина Васильевна, кандидат филологических наук, доцент кафедры иностранных языков Ростовский государственный университет путей сообщения (2. Ростов-на-Дону, Российская Федерация)

Аннотация. В статье обосновывается необходимость предъявления новых требований к подготовке профессиональных кадров. Подчеркивается роль иностранных языков в процессе обучения в высших учебных заведениях, что отражено в федеральных государственных образовательных стандартах. В данном исследовании рассмотрены подходы и особенности формирования компетентностной модели обучения деловому общению на иностранном языке на примере магистратуры железнодорожного вуза. Данная модель представляется как комплекс лингвистических, коммуникативных, культурологических, психологических и профессиональных компонентов. Исследуются особенности процесса обучения иностранному языку в магистратуре технических вузов. Изучаются основания для обучения деловому иностранному языку с помощью междисциплинарного подхода. Междисциплинарный подход позволяет интегрировать знания и умения обучающихся, приобретенные ими в процессе изучения других дисциплин и успешно применяемые для освоения иностранного языка и формирования компетенции иноязычного делового общения. Представлены учебные пособия, разработанные преподавателями кафедры иностранных языков Ростовского государственного университета путей сообщения для обучающихся технических направлений подготовки магистратуры. Приведены примеры учебных заданий и некоторые технологии обучения, применяемые автором в процессе реализации рабочих программ в магистратуре железнодорожного вуза. Определены перспективные направления дальнейших исследований.

Ключевые слова: магистратура технических вузов; железнодорожный вуз; компетенция делового общения на иностранном языке; деловой немецкий язык; междисциплинарный подход; учет лингвокультурологических особенностей; учебно-методическое пособие для обучающихся магистратуры; интерактивные технологии при обучении деловому общению на иностранном языке; специальный текст; презентационная речь; симуляция.

Современные процессы всемирной глобализации и интенсификации вхождения России в мировое пространство настоятельно требуют владения иностранным языком как неотъемлемой составляющей общей профессиональной компетенции выпускников вузов. Решающее значение в этой связи имеет подготовка высококвалифицированных кадров по магистерским программам, основная цель которых заключается в формировании у обучающихся способности общения на межкультурном уровне в профессиональной сфере. Поэтому вполне обоснованной является выработка новых требований, предъявляемых к подготовке профессиональных кадров, а соответственно, и к формированию профессиональной компетентности, дающей возможность реализовать все виды деятельности (организационную, управленческую, исследовательскую) в выбранной профессии и успешное профессиональное общение на иностранном языке 